\title{
3-5 BI-RADs Microcalcifications: Correlation between MRI and Histological Findings
}

\author{
Valeria Fiaschetti, Chiara Adriana Pistolese, Tommaso Perretta, Elsa Cossu, Chiara Arganini, \\ Claudia Salimbeni, Angela Lia Scarano, Silvia Arduini, and Giovanni Simonetti
}

Department of Diagnostic Imaging, Molecular Imaging, Interventional Radiology and Radiotherapy,
University of Tor Vergata, Viale Oxford 81, 00133 Rome, Italy

Correspondence should be addressed to Chiara Adriana Pistolese, chiarapistolese@uniroma2.it

Received 14 April 2011; Accepted 12 May 2011

Academic Editor: E. A. Rakha

Copyright ( $\odot 2011$ Valeria Fiaschetti et al. This is an open access article distributed under the Creative Commons Attribution License, which permits unrestricted use, distribution, and reproduction in any medium, provided the original work is properly cited.

\begin{abstract}
Purpose. To evaluate the correlation between MRI and histopathological findings in patients with mammographically detected 3-5 BI-RAD (Breast Imaging Reporting And Data Systems) microcalcifications and to allow a better surgical planning. Materials and Method. 62 female Patients (age $50 \pm 12$ ) with screening detected 3-5 BI-RAD microcalcifications underwent dynamic 3 T contrast-enhanced breast MRI. After 30-day (range 24-36 days) period, 55 Patients underwent biopsy using stereotactic vacuumassisted biopsy (VAB), 5 Patients underwent stereotactic mammographically guided biopsy, and 2 Patients underwent MRI-guided VAB. Results. Microhistology examination demonstrated 36 malignant lesions and 26 benign lesions. The analysis of MRI findings identified 8 cases of MRI BI-RADS 5, 23 cases of MRI BI-RADS 4, 11 cases of MRI BI-RADS 3, 4 cases type A and 7 cases type B, and 20 cases of MRI BI-RADS 1-2. MRI sensitivity, specificity, positive predictive value, and negative predictive value were $88.8 \%$, $76.9 \%, 84.2 \%$, and $83.3 \%$, respectively.
\end{abstract}

\section{Introduction}

Mammographically detected microcalcifications are early diagnosed breast cancers, found in approximately $70 \%$ of ductal carcinoma in situ (DCIS) [1,2]. During the last 20 years, the prevalence of DCIS has grown from less than $5 \%$, before the start of mammographic screening, to $15 \%-$ $30 \%$ in women regularly checked with mammography [3]. The extension of screening mammography has resulted in a decreased number of patients who die of breast cancer, because mammography is sensitive for the detection of clinically occult breast cancer $[4,5]$. Mammography has high sensitivity and low specificity, the positive predictive value (PPV) being 15\%-30\% for malignant nonpalpable lesions [3]. The sensitivity of contrast-enhanced MR imaging in detecting invasive breast cancers has been extensively shown to be very high (94-100\%), with a specificity of approximately $65-80 \%$ [6]. Conversely, until now, there is no agreement regarding the sensitivity and specificity of breast MRI for the detection of in situ ductal cancers, and the role of MRI in characterizing breast microcalcifications remains a debated issue [7]. In fact the reported values of sensitivity range between $45 \%$ and $100 \%$ and the specificity between $37 \%$ and $95 \%$ [8]. A recent study suggested that nonpalpable lesions with microcalcifications categorized as BI-RADS 3 should undergo a biopsy procedure until a more reliable system for the description and classification of microcalcifications is available [9]. $\mathrm{VAB}$ has a sufficient sensitivity and specificity to replace surgical biopsy and offers valuable advantages for the evaluation of small concerning lesions and microcalcifications [10].

The purpose of our study was to determine the frequency of malignancy in BI-RADS 3-5 microcalcifications using dynamic contrast-enhanced MR imaging findings in screening detected microcalcification lesions and its correlation with histopathological findings. 


\section{Materials and Method}

2.1. Study Population. From January 2007 to December 200962 women with BI-RADS 3-5 microcalcifications on mammography underwent breast MRI before a stereotactic biopsy using vacuum-assisted biopsy (VAB). In 8/62 patients the microcalcifications were associated with an opacity, and in $1 / 62$ patients the microcalcifications were associated with glandular distortion. The study received ethics committee approval, and all patients provided informed consent after a clear explanation of the benefits and potential risks.

2.2. Mammography. Digital mammographic examinations were performed with GE Senographe DS (General Electric, Milwaukee, USA); mammographic magnification was performed using the view that allowed the best visualisation of the microcalcifications, and the microcalcification extension was evaluated using the standard views.

2.3. Mammography Interpretations. The digital mammograms acquired were analyzed in a blinded fashion by two expert radiologists. All cases of microcalcifications were classified according to the method proposed by American College of Radiology [11], and only those classified as BIRADS 3-5 were selected.

2.4. MRI. MR imaging was performed in all cases before microhistology. Dynamic MRI was performed during the 7-14th day of the menstrual period in fertile woman. The instrument was a $3.0 \mathrm{~T}$ (Achieva, Philips Healthcare, Best, The Netherlands) MRI apparatus, equipped with 4 channels reception dedicated coil. All the subjects underwent MRI exam with SENSE technology. Patients were examined in prone position with both breasts positioned inside the coil. MRI images were acquired on axial planes. After a survey acquisition, MRI protocol consisted of the following sequences: T1 (TSE) (TR/TE $6.8 / 3.3 \mathrm{~ms}$; thickness, $3 \mathrm{~mm}$, gap 0; matrix, $512 \times 512$ ), T2-TSE (TR/TE 3800/140 ms; thickness, $3 \mathrm{~mm}$, gap 0; matrix, $225 \times 512)$, a short tau inversion recovery sequence (STIR) (TR/TE/TI/4,000/42/155 ms; $3.0 \mathrm{~mm}$, gap $0 ; 320 \times 224)$, and a T1 dynamic sequence $(2 \mathrm{D})$ (FFE) (TR/TE 290/4.6 ms; flip angle, $90^{\circ}$; matrix $256 \times 512$; thickness, $3 \mathrm{~mm}$; 8 dynamics; with $50 \mathrm{~s}$ time resolution for each). The T1 dynamic sequences were acquired by previous $0.1 \mathrm{mmol} / \mathrm{Kg}$ gadolinium bolus injection (gadopentetic acid and dimeglumine salt, Magnevist; Schering, Berlin, Germany) administered with a $2 \mathrm{~mL} / \mathrm{sec}$ flux, followed by a saline flush of $20 \mathrm{~mL}$.

2.5. MR Images Analysis. Any contrast enhancement in the area of intermediate microcalcification was considered positive (true positive). The absence of contrast enhancement in the area of intermediate microcalcification was considered negative. All exams were analysed by two radiologists experienced in interpreting breast MRI. During the assessment of MRI exams, the images of the mammography previously performed were available. Each observer assessed the enhancement characteristics, like: shape, nodular (assessment of shape: circular, oval, lobulated, or irregular; edges assessment: smooth, irregular, spiculated) or nonnodular (distribution pattern assessment: nodular, linear/segmental, dendritic). As to the evaluation of the signal/time intensity ratio $(I s / t)$, regions of interests (ROI) included in the suspect lesions were manually outlined inside the major enhancement areas. The $I s / t$ curves were characterized depending on the presence of persistent enhancement: type (1) with a continuous increase in signal intensity on each successive contrast-enhanced image; type (2) "plateau pattern" in which an initial increase in signal intensity was followed by a flattening and fluctuation of the enhancement curve; type (3) "washout pattern," with an initial increase and subsequent decrease in signal intensity. Results of the MRI study were classified into five BIRADS categories: MRI 1-2 negative/benign, MRI 3a probably benign lesion, and MRI 3 b borderline lesion/probably malignant; BIRADS 4-5: probably malignant/malignant [11]. The so-called borderline breast lesions may lie on a spectrum of pathological entities which are difficult to distinguish from malignant lesions $[2,3]$. The borderline lesions recognised in this study are atypical ductal hyperplasia, flat atypia adenosis, and ductal papilloma. Actually a MRI demonstrated that borderline lesion is referred for a diagnostic surgical biopsy to exclude the presence of a nearly carcinoma lesion.

2.6. VAB System. VAB VACORA (BARD) system was adopted to carry out most parts of our biopsies. The system is equipped with a MRI compatible, disposable coaxial stainless steel introducer needed to guide the a-magnetic fine needle up to the area of interest. The needle consists of two stainless steel cannula with 10 Gauge outer diameter; the inner cannula includes a window for the sample collection, connected with the aspiration cylinder. The VACORA BARD system can be employed as handheld device as well; it allows pressure vacuum generation through an electric engine equipped with a microprocessor. One radiologist with 6 year of prior experience in VAB performed the biopsy with the patients prone on a digital stereotactic table. Complete or partial removal of the microcalcifications was assessed in all cases on two-view full-field mammograms immediately. If microcalcifications had been removed completely or almost completely, clips were placed through the 10-gauge probe to identify the VAB site for sequent surgical excision $[12,13]$. The histopathological result was correlated with the mammographic findings by both the radiologist and pathologist in all cases.

2.7. Histological Diagnosis. The histological findings were classified into two groups, malignant and benign. Malignant lesions included infiltrative ductal cancer, ductal in situ cancer (DCIS), and high-risk lesions as atypical ductal hyperplasia, flat atypia, and ductal papilloma. Lesions not classified as malignant and high-risk lesions were identified as benign lesions. 
TABLE 1: Mx BI-RADS and histological correlation.

\begin{tabular}{lll}
\hline MX BI-RADS (N lesions) & Histology, benign lesions & Histology, malignant lesions \\
\hline & 8 apocrine metaplasia & 1 DCIS \\
$3(23)$ & 6 typical ductal hyperplasia & 1 flat atypia \\
& 2 sclerosing adenosis & 3 atypical ductal hyperplasia \\
& & 2 ductal papilloma \\
\hline \multirow{3}{*}{$4(32)$} & 5 apocrine metaplasia & 20 DCIS \\
& 4 typical ductal hyperplasia & 1 infiltrative ductal cancer \\
& 1 sclerosing adenosis & 1 ductal papilloma \\
\hline $5(7)$ & & 1 DCIS \\
& & 6 infiltrative ductal cancer \\
\hline
\end{tabular}

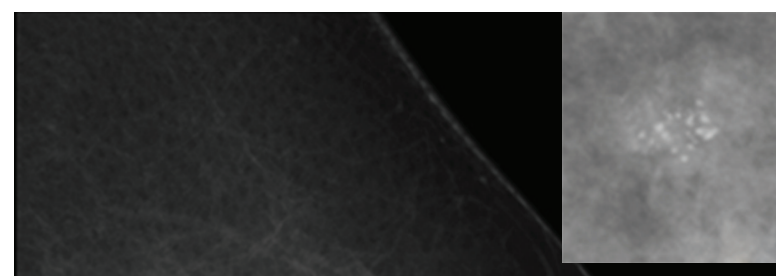

(b)

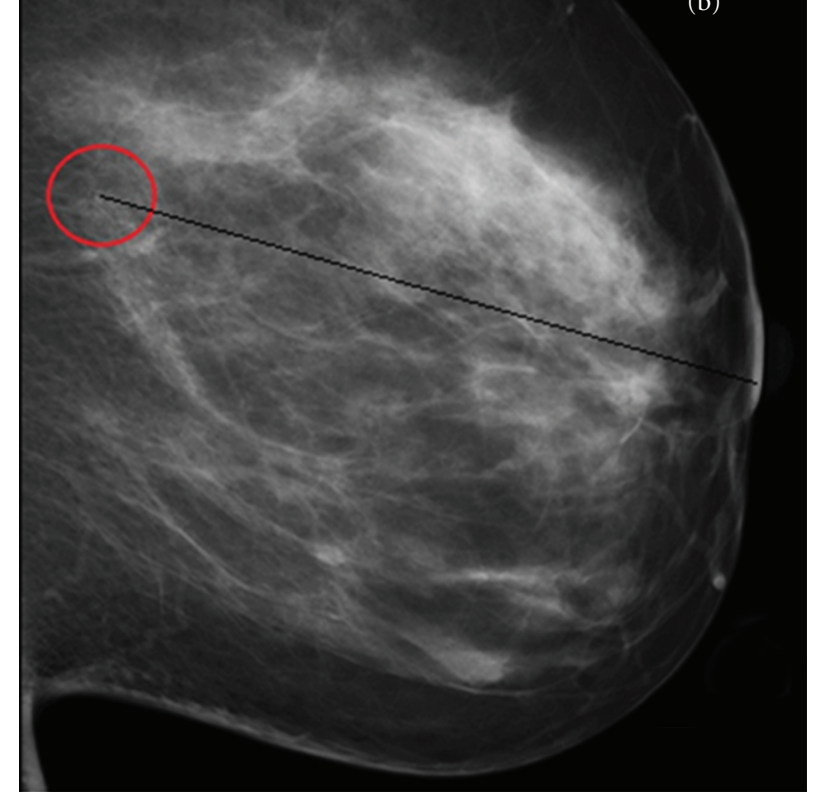

(a)

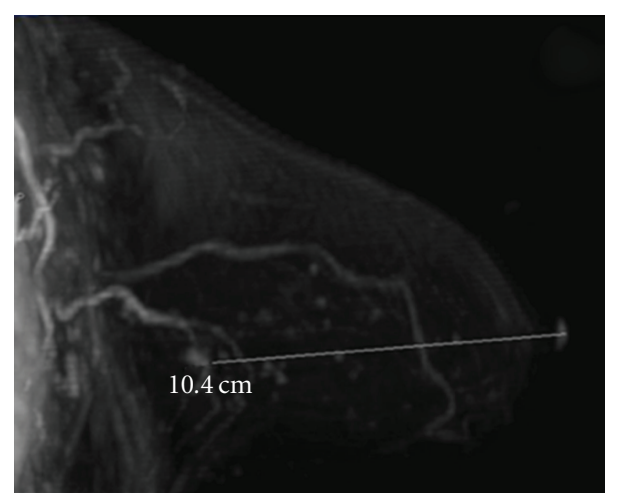

(c)

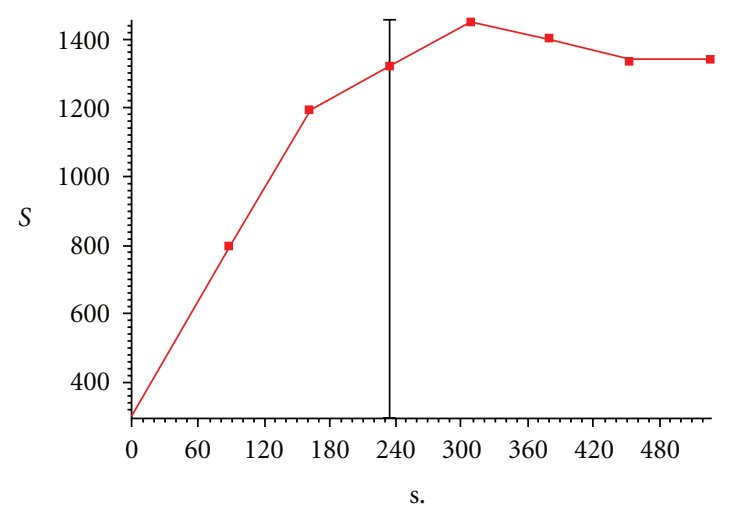

(d)

Figure 1: (a-b) A case of MX BI-RADS 4; mammography shows a cluster of microcalcifications in deep upper-outer quadrants of the left breast. (c) MRI shows an irregular area with irregular and segmented contrast-enhancement with longitudinal development (about 15 mm) in deep retroareolar region, next to the cluster of microcalcification seen by Mammography; this case has been classified as MRI BI-RADS 4. (d) time-intensity curve documents heavy but not fast washin and slower washout of contrast-enhancement. Histology demonstrated a DCIS.

\section{Results}

Microhistology examination demonstrated 36 malignant lesions and 26 benign lesions. In particular of 36 malignant lesions, 7 were described as infiltrative ductal cancer, 22 as ductal in situ cancer (DCIS), 3 as atypical ductal hyperplasia, 1 as flat atypia, and 3 as ductal papilloma; on the other hand the 26 benignant lesions were represented by 13 apocrine metaplasia, 10 typical ductal hyperplasia and, 3 sclerosing adenosis. The mammographic examination of 62 Patients showed 26 cases of BI-RADS 3, 29 cases of BI-RADS 4, and 7 cases of BI-RADS 5. In 8 patients the microcalcifications were associated with an opacity while in 1 patient the microcalcifications were associated with glandular distortion. The correlation between mammography and histology results demonstrated that of 23 cases of BI-RADS 3, 8 were 


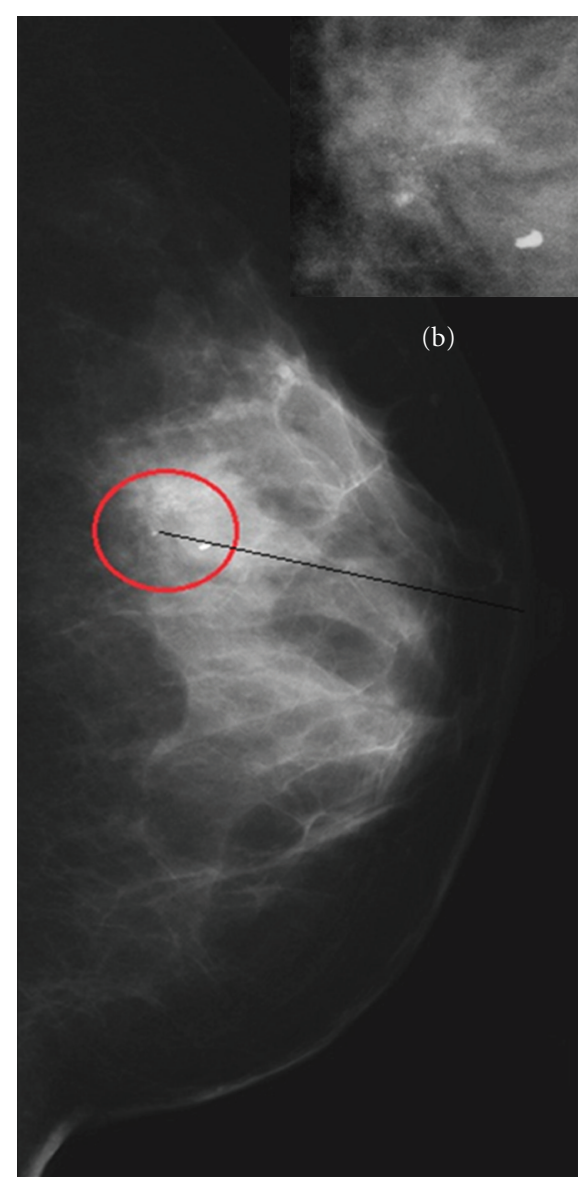

(a)

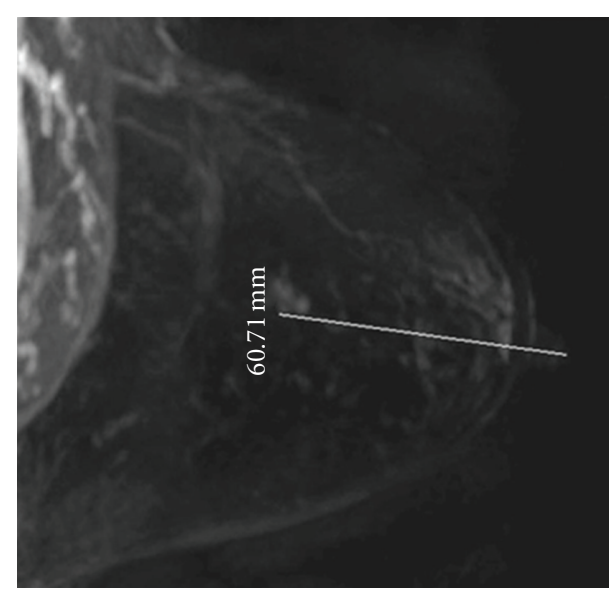

(c)

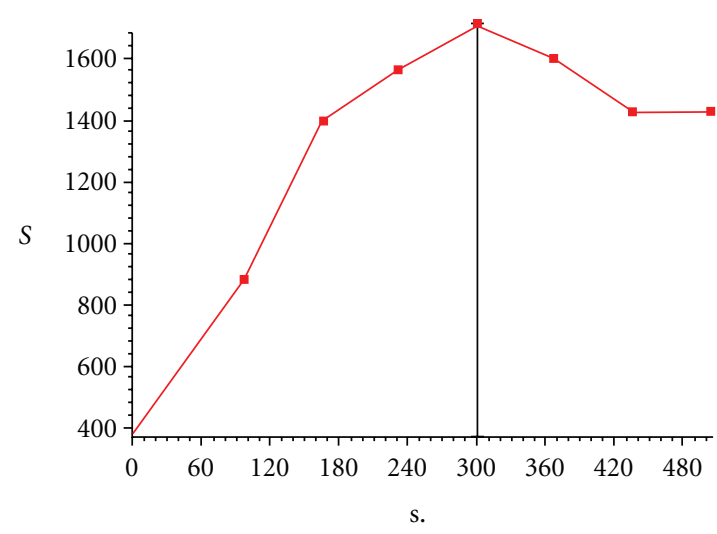

(d)

FIgURE 2: (a-b) A case of MX BI-RADS 3; mammography shows a cluster of microcalcifications in upper quadrants of left breast. (c) MRI shows a millimetric pseudonodular contrast-enhancement without sure substratum in morphologic sequences in the same region of Mx microcalcifications; it has been classified as MRI BI-RADS 4. (d) The time-intensity curve has heavy but not fast washin and subsequent plateau of contrast-enhancement. Histology conversely demonstrated a sclerosing adenosis.

apocrine metaplasia, 6 were typical ductal hyperplasia, 2 were sclerosing adenosis, 1 were DCIS, 1 was flat atypia, 3 atypical ductal hyperplasia, and 2 ductal papilloma. Of 32 cases of BI-RADS 4, 5 were apocrine metaplasia, 4 typical ductal hyperplasia, 1 was sclerosing adenosis, 20 DCIS, 1 infiltrative ductal cancer, and 1 ductal papilloma; of 7 cases of BI-RADS 5, 1 was DCIS and 6 were infiltrative ductal cancer (Table 1). Mammographic sensitivity, specificity, positive predictive value, and negative predictive value were $80.5 \%$, $61.5 \%, 74.3 \%$, and $69.5 \%$, respectively. Including only the DCIS findings, we found that mammographic sensitivity, specificity, positive predictive value, and negative predictive value were $95.4 \%, 61.5 \%, 67.7 \%$, and $94.1 \%$, respectively.

The analysis of MRI findings identified 8 cases of MRI BI-RADS 5, 23 cases of MRI BI-RADS 4, 11 cases of MRI BI-RADS 3, 4 cases type A and 7 cases type B, and 20 cases of MRI BI-RADS 1-2. The correlation between MRI and histology results showed that of 8 MRI BI-RADS 5, 7 cases were confirmed as infiltrative ductal cancer and 1 as DCIS; of 23 MRI BI-RADS 4, 17 were confirmed by histology as DCIS (Figure 1), whereas 1 were described as apocrine metaplasia,
3 as typical ductal hyperplasia, and 2 as sclerosing adenosis (Figure 2); of MRI BI-RADS 3 type A, 1 was confirmed as apocrine metaplasia, 1 as typical ductal hyperplasia, and 1 as sclerosing adenosis and 1 ductal papilloma; of MRI BI-RADS 3 type B, 2 were confirmed as atypical ductal hyperplasia ad 1 as flat atypia, 2 as DCIS, and 2 ductal papilloma; furthermore of 20 cases of MRI BI-RADS 1-2, 17 were confirmed by histology as 11 apocrine metaplasia and 6 as typical ductal hyperplasia, while 2 were revealed to be DCIS (Figure 3 ) and 1 to be an atypical ductal hyperplasia (Table 2). MRI sensitivity, specificity, positive predictive value, and negative predictive value were $88.8 \%, 76.9 \%, 84.2 \%$, and $83.3 \%$, respectively. Including only the DCIS findings, we found that the MRI sensitivity, specificity, positive predictive value, and negative predictive value were $90.9 \%, 76.9 \%, 76.9 \%$, and $90.9 \%$, respectively.

Of 62 patients, 18 (29\%) did not show any kind of contrast enhancement at MRI, while 44 (71\%) showed contrast enhancement; in particular 7 presented a dendritic enhancement, 18 a linear or segmental enhancement, and 19 a nodular enhancement (Table 3 ). 


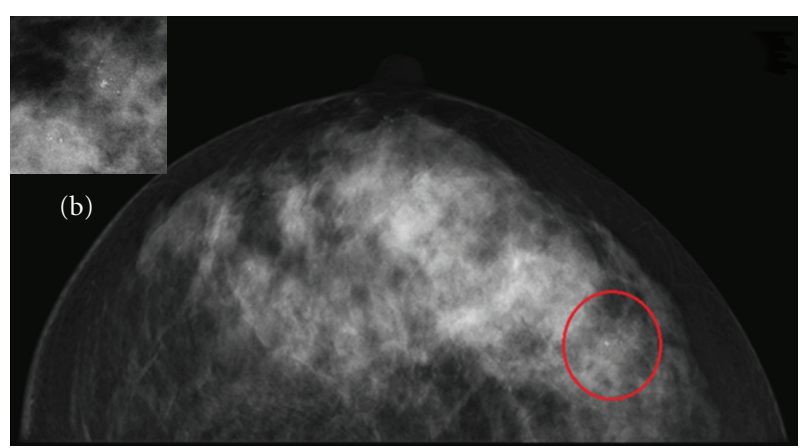

(a)

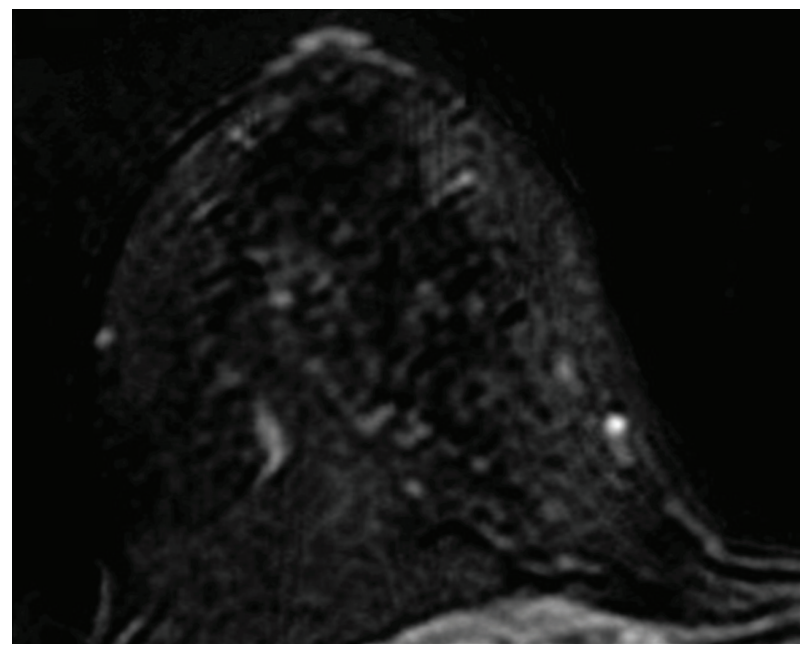

(c)

Figure 3: (a-b) A case of MX BI-RADS 4; mammography shows a cluster of microcalcifications in upper-outer quadrants of the right breast. (c) This case has been classified as MRI BI-RADS 1-2 because there was not contrast-enhancement in upper-outer quadrants of the right breast. Histology demonstrated a DCIS.

On Table 4 we analysed the time intensity curve correlated with the MRI BI-RAD and we showed that of patients with contrast enhancement, 6/44 had a type 1,16/44 a type 2 , and 22/44 a type 3 .

Comparing MX and MRI results we found that 2 cases of MX BI-RADS 4, identified subsequently as DCIS, were described as MRI BI-RADS 1-2, because they did not show contrast enhancement (Figure 3 ).

Despite that, with regard to disease extension, we found that mammography underestimation existed in 6/29 cases (20.9\%), confirmed later by histology; in particular of 7 infiltrative ductal cancer histologically detected, MRI permitted to visualise 2 cases of multifocality and 1 case of multicentricity, whereas of 22 DCIS, MRI visualised 2 cases of multifocality and 1 case of multicentricity.

\section{Discussion}

Mammography is extremely sensitive in detecting microcalcifications even though it does not permit to distinguish malignant from benign lesions and invasive carcinoma from DCIS [14]. In fact in our population sensitivity of mammography in detection of cancer and early cancer related to microcalcifications was $80.5 \%$, and specificity was only $61.5 \%$. The presence of microcalcifications on mammography is often referred to early diagnosed breast cancers and is found in approximately $70 \%$ of minimal breast cancers and frequently in DCIS [15]. Stomper and Margolin reports that mammographically detected microcalcifications are the only sign in $72 \%$ of clinically occult DCIS lesions [15]. The low specificity of microcalcifications as a feature of malignancy, $10-70 \%$ according to literature, is histologically demonstrated in a high number of diagnostic biopsies [14]. On the other hand, approximately $75 \%$ of lesions that are detected, suspected, or indeterminate on mammography are revealed to be benign at biopsy [16], suggesting that many biopsies are performed unnecessarily because the indication for VAB has not yet been fully established.

Dynamic contrast-enhanced MRI is an effective diagnostic technique for symptomatic breast diseases. However its role in evaluating clinically occult disease associated with mammographically detected suspicious microcalcifications has to be clarified. Mammography or ultrasound cannot be replaced by breast MRI even when it is indicated to perform biopsy. Nevertheless contrast-enhanced dynamic breast MRI is actually the best technique in the detection of multifocality or bilateral incidence of carcinoma mainly in dense type of breast. In our study mammography underestimated disease extension in 6 cases $(20.9 \%)$, in particular of 7 infiltrative ductal cancer histologically detected, MRI permitted to visualise 2 cases of multifocality and 1 case of multicentricity, whereas of 22 DCIS, MRI visualised 2 cases of multifocality and 1 case of multicentricity. Knowing that allowed a better surgical planning in these patients. Previous MR studies have reported variable accuracy of MRI for classification of microcalcifications $[17,18]$. Early studies suggested that dynamic contrast-enhanced MRI should not be used to assess microcalcifications $[19,20]$ because MRI is unable to identify small calcifications, which are typically associated with malignant disease. Westerhof et al. [19] reported a sensitivity of $45 \%$ and a specificity of $72 \%$ for dynamic MRI in patients with mammographically suspicious microcalcifications. In another study, Gilles et al. [20] observed a sensitivity of $95 \%$ and a specificity of only $51 \%$ for MRI; in this case, specificity was potentially lower because the presence or absence of contrast uptake in the breast was the only parameter used to define the malignancy. The latest studies have a better rate of diagnosis due to technological improvements. Bazzocchi et al. [17] reported that MRI using up-to-date 3D sequences and combined morphologicalkinetic evaluation had a sensitivity of $87 \%$, a specificity of $68 \%$, and an accuracy of $80 \%$. The high variability of MRI diagnostic accuracy in evaluating microcalcifications reported in these studies is related to the use of different criteria as lesion size, histological variability of cancer, cancer angiogenesis, type of enhancement, enhancement pattern, distribution in the breast, margins (regular, irregular), and use of different pulse sequences and scan planes [20]. Cilotti et al. in an analysis of morphological and dynamic features of BIRADS 3-5 microcalcifications observed a sensitivity of 
TABLE 2: MRI BI-RADS and histological correlation.

\begin{tabular}{|c|c|c|}
\hline MRI BI-RADS ( $N$ lesions) & Histology, benign lesions & Histology, malignant lesions \\
\hline \multirow{2}{*}{$1-2(20)$} & 11 apocrine metaplasia & 2 DCIS \\
\hline & 6 typical ductal hyperplasia & 1 atypical ductal hyperplasia \\
\hline \multirow{3}{*}{$3 \mathrm{~A}(4)$} & 1 apocrine metaplasia & \\
\hline & 1 typical ductal hyperplasia & 1 ductal papilloma \\
\hline & 1 sclerosing adenosis & \\
\hline \multirow{4}{*}{ 3B (7) } & & 2 atypical ductal hyperplasia \\
\hline & & 1 flat atypia \\
\hline & & 2 DCIS \\
\hline & & 2 ductal papilloma \\
\hline \multirow{3}{*}{$4(23)$} & 1 apocrine metaplasia & \\
\hline & 3 typical ductal hyperplasia & 17 DCIS \\
\hline & 2 sclerosing adenosis & \\
\hline \multirow{2}{*}{$5(8)$} & & 7 infiltrative ductal cancer \\
\hline & & 1 DCIS \\
\hline
\end{tabular}

TABLE 3: MRI enhancement.

\begin{tabular}{|c|c|c|c|c|}
\hline \multirow{2}{*}{ MRI BI-RADS } & \multicolumn{3}{|c|}{ Enhancement } & \multirow{2}{*}{ No enhancement } \\
\hline & Dendritc & Linear/segmental & Nodular & \\
\hline $1-2$ & & & 2 & 18 \\
\hline $3 \mathrm{~A}$ & & & 4 & \\
\hline $3 \mathrm{~B}$ & & 3 & 4 & \\
\hline 4 & & 14 & 9 & \\
\hline 5 & 7 & 1 & & \\
\hline
\end{tabular}

TABLE 4: MRI time-intensity curve of enhancement.

\begin{tabular}{lccc}
\hline MRI BI-RADS & Type 1 & $\begin{array}{c}\text { Time intensity curve } \\
\text { Type 2 }\end{array}$ & Type 3 \\
\hline $1-2$ & 2 & & \\
3A & 4 & 6 & 1 \\
3B & & 10 & 13 \\
4 & & & 8 \\
5 & & & \\
\hline
\end{tabular}

MRI of $73 \%$. They considered MRI BIRADS categories 1, 2 , and 3 as benign, according to histopathological diagnosis and 4 and 5 as malignant [21]. In our study, the sensitivity of contrast-enhanced MRI was higher $(88.8 \%$ versus $73 \%$ in Cilotti's study). Compared with previous studies, our study could show better results, in addition to the use of up-to-date 3D sequences, probably because of the revised MRI classification system. In fact we proposed a more detailed definition of BIRADS 3 enhancement to detect border-line lesions such as atypical ductal hyperplasia, flat atypia, and ductal papilloma. In our study we observed that in MRI BI-RADS 3 the angiogenesis gave in 8 cases a nodular enhancement and in 3 cases a linear/segmental enhancement. Furthermore we noticed that in 4 cases the time-intensity curve was type 1 , in 6 cases was type 2 while in one case was type 3 . So correlating the type of enhancement and its time-intensity curve with histological findings, we classified MRI BI-RADS 3 in two subgroups (MRI BI-RADS $3 \mathrm{~A}$ and MRI BI-RADS
3B). In fact, in 3 cases of MRI BI-RADS 3A the histology revealed 3 benign lesions, and in 5 cases of MRI BI-RADS $3 \mathrm{~B}$ histology revealed border-line lesions (2 atypical ductal hyperplasia, 1 flat atypia, and 2 ductal papilloma). The socalled border-line breast lesions are lesions which are difficult to distinguish from malignant lesions [22-24]. They are represented by ductal papilloma, atypical ductal hyperplasia, and flat atypia. As defined by the World Health Organization (WHO) Working Group on Pathology and Genetics of Tumors of the Breast [25], epithelial atypia is divided into atypical ductal hyperplasia, flat epithelial atypia (or DIN 1a). Due to a lack of standardized terminology, atypical ductal hyperplasia and flat atypia are sometimes poorly differentiated on pathological examination [26]. As defined by the WHO $[23,24]$, FEA is an "intraductal alteration characterized by replacement of the native epithelial cells by a single or 3-5 layers of mildly atypical cells". However the guide-lines consider to treat the borderline lesions as an 
early breast cancer and recommend surgical excision. Paying attention to these kind of borderline lesions permits to avoid the transformation of a small lesion into an invasive cancer. In conclusion breast MRI could change the prognosis of the breast cancer detecting the border-line lesions that are recently defined, to be more precise, the precursors of the early breast cancer.

\section{Conclusions}

In conclusion, our study has revealed that contrast-enhanced breast MRI have a better sensitivity and specificity in the evaluation of the early breast cancer related to mammographically detected microcalcifications, than other studies. In fact in our experience breast MRI sensitively improved the percentage of diagnosis of malignancy in mammographically detected microcalcification. This is due to the use of different criteria as lesion size, histological variability of cancer, cancer angiogenesis, type and pattern of enhancement, margins, and use of 3D sequences but specially to a revision of MRI BI-RADS classification, considering the border-line lesions as proposed by the World Health Organization (WHO) Working Group on Pathology and Genetics of Tumors of the Breast.

\section{References}

[1] P. C. Stomper, J. Geradts, S. B. Edge, and E. G. Levine, "Mammographic predictors of the presence and size of invasive carcinomas associated with malignant lesions without a mass," American Journal of Roentgenology, vol. 181, no. 6, pp. 1679-1684, 2003.

[2] B. Barreau, I. De Mascarel, C. Feuga et al., "Mammography of ductal carcinoma in situ of the breast: review of 909 cases with radiographic-pathologic correlations," European Journal of Radiology, vol. 54, no. 1, pp. 55-61, 2005.

[3] S. G. Orel, M. H. Mendonca, C. Reynolds, M. D. Schnall, L. J. Solin, and D. C. Sullivan, "MR imaging of ductal carcinoma in situ," Radiology, vol. 202, no. 2, pp. 413-420, 1997.

[4] D. A. Berry, K. A. Cronin, S. K. Plevritis et al., "Effect of screening and adjuvant therapy on mortality from breast cancer," New England Journal of Medicine, vol. 353, no. 17, pp. 1784-1792, 2005.

[5] L. L. Humphrey, M. Helfand, B. K. S. Chan, and S. H. Woolf, "Breast cancer screening: a summary of the evidence for the U.S. Preventive Services Task Force," Annals of Internal Medicine, vol. 137, no. 5, pp. 347-360, 2002.

[6] S. H. Heywang-Kobrunner, "Contrast-enhanced magnetic resonance imaging of the breast," Investigative Radiology, vol. 29, no. 1, pp. 94-104, 1994.

[7] P. Viehweg, D. Lampe, J. Buchmann, and S. H. HeywangKöbrunner, "In situ and minimally invasive breast cancer: morphologic and kinetic features on contrast-enhanced MR imaging," Magnetic Resonance Materials in Physics, Biology and Medicine, vol. 11, no. 3, pp. 129-137, 2000.

[8] C. K. Kuhl, "MRI of breast tumors," European Radiology, vol. 10, pp. 46-58, 2000.

[9] R. M. Pijnappel, P. H. M. Peeters, J. H. C. L. Hendriks, and W. P. T. M. Mali, "Reproducibility of mammographic classifications for non-palpable suspect lesions with microcalcifications," British Journal of Radiology, vol. 77, no. 916, pp. 312-314, 2004.
[10] E. Fondrinier, G. Lorimier, V. Guerin-Boblet, A. F. Bertrand, C. Mayras, and N. Dauver, "Breast microcalcifications: multivariate analysis of radiologic and clinical factors for carcinoma," World Journal of Surgery, vol. 26, no. 3, pp. 290-296, 2002.

[11] American College of Radiology (ACR). Breast Imaging Reporting and Data System Atlas (BI-RADS Atlas), 2011.

[12] L. Liberman, D. D. Dershaw, E. A. Morris, A. F. Abramson, C. M. Thornton, and P. P. Rosen, "Clip placement after stereotactic vacuum-assisted breast biopsy," Radiology, vol. 205, no. 2, pp. 417-422, 1997.

[13] C. A. Pistolese, A. M. Ciarrapico, F. Della Gatta et al., "Costeffectiveness analysis of two vacuum-assisted breast biopsy systems: mammotome and Vacora," Radiologia Medica, vol. 114, no. 5, pp. 743-756, 2009.

[14] L. W. Bassett, "Mammographic analysis of calcifications," Radiologic Clinics of North America, vol. 30, no. 1, pp. 93-105, 1992.

[15] P. C. Stomper and F. R. Margolin, "Ductal carcinoma in situ: the mammographer's perspective," American Journal of Roentgenology, vol. 162, no. 3, pp. 585-591, 1994.

[16] G. Pfarl, T. H. Helbich, C. C. Riedl et al., "Stereotactic II-gauge vacuum-assisted breast biopsy: a validation study," American Journal of Roentgenology, vol. 179, no. 6, pp. 1503-1507, 2002.

[17] M. Bazzocchi, C. Zuiani, P. Panizza et al., "Contrast-enhanced breast MRI in patients with suspicious microcalcifications on mammography: results of a multicenter trial," American Journal of Roentgenology, vol. 186, no. 6, pp. 1723-1732, 2006.

[18] J. Zhu, Y. Kurihara, Y. Kanemaki et al., "Diagnostic accuracy of high-resolution MRI using a microscopy coil for patients with presumed DCIS following mammography screening," Journal of Magnetic Resonance Imaging, vol. 25, no. 1, pp. 96-103, 2007.

[19] J. P. Westerhof, U. Fischer, J. D. Moritz, and J. W. Oestmann, "MR imaging of mammographically detected clustered microcalcifications: is there any value?" Radiology, vol. 207, no. 3, pp. 675-681, 1998.

[20] R. Gilles, M. Meunier, O. Lucidarme et al., "Clustered breast microcalcifications: evaluation by dynamic contrast-enhanced subtraction MRI," Journal of Computer Assisted Tomography, vol. 20, no. 1, pp. 9-14, 1996.

[21] A. Cilotti, C. Iacconi, C. Marini et al., "Contrast-enhanced MR imaging in patients with BI-RADS 3-5 microcalcifications," Radiologia Medica, vol. 112, no. 2, pp. 272-286, 2007.

[22] T. W. Jacobs, J. L. Connolly, and S. J. Schnitt, "Nonmalignant lesions in breast core needle biopsies: to excise or not to excise?" American Journal of Surgical Pathology, vol. 26, no. 9, pp. 1095-1110, 2002.

[23] N. Houssami, S. Ciatto, M. Bilous, V. Vezzosi, and S. Bianchi, "Borderline breast core needle histology: predictive values for malignancy in lesions of uncertain malignant potential (B3)," British Journal of Cancer, vol. 96, no. 8, pp. 1253-1257, 2007.

[24] S. Heywang-Kobrunner and R. Beck, Contrast-Enhanced MRI of the Breast, Springer, Berlin, Germany, 2nd edition, 1995.

[25] F. A. Tavassoli, R. R. Millis, W. Boecker, and S. R. Lakhani, "Lobular neoplasia," in Tumors of the Breast and Female Genital Organs, F. A. Tavassoli and P. Devilee, Eds., World Health Organization Classification of Tumours, pp. 60-64, IARC press, Lyon, France, 2003.

[26] M. M. Guerra-Wallace, W. N. Christensen, and R. L. White, "A retrospective study of columnar alteration with prominent apical snouts and secretions and the association with cancer," American Journal of Surgery, vol. 188, no. 4, pp. 395-398, 2004. 


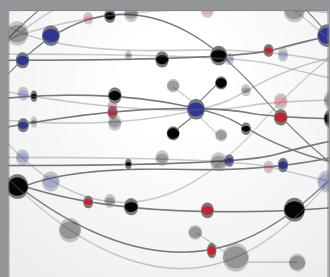

The Scientific World Journal
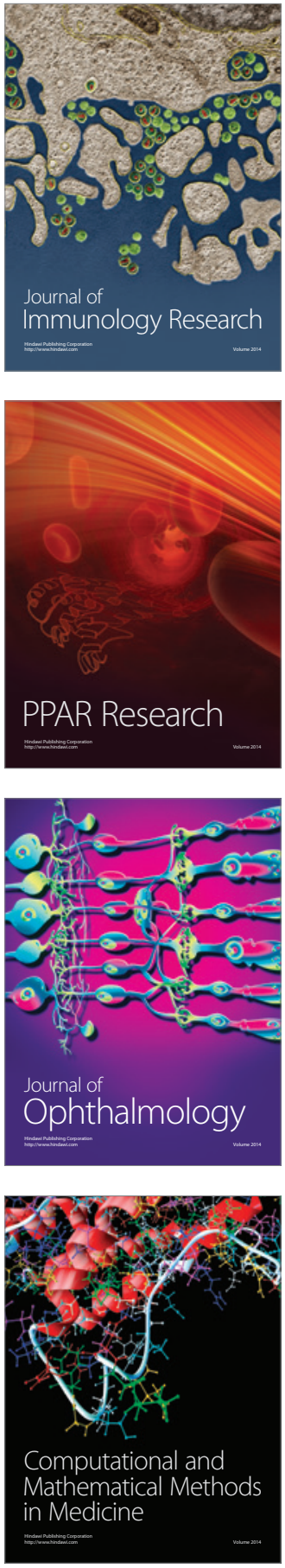

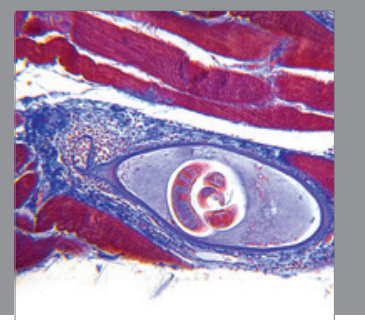

Gastroenterology

Research and Practice
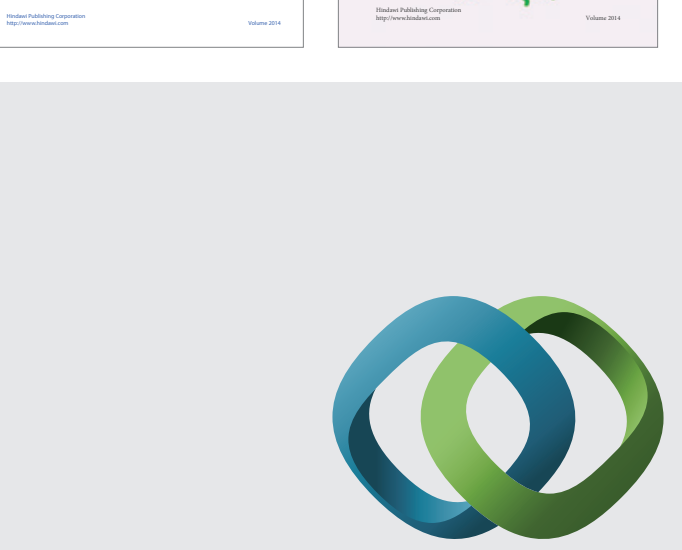

\section{Hindawi}

Submit your manuscripts at

http://www.hindawi.com
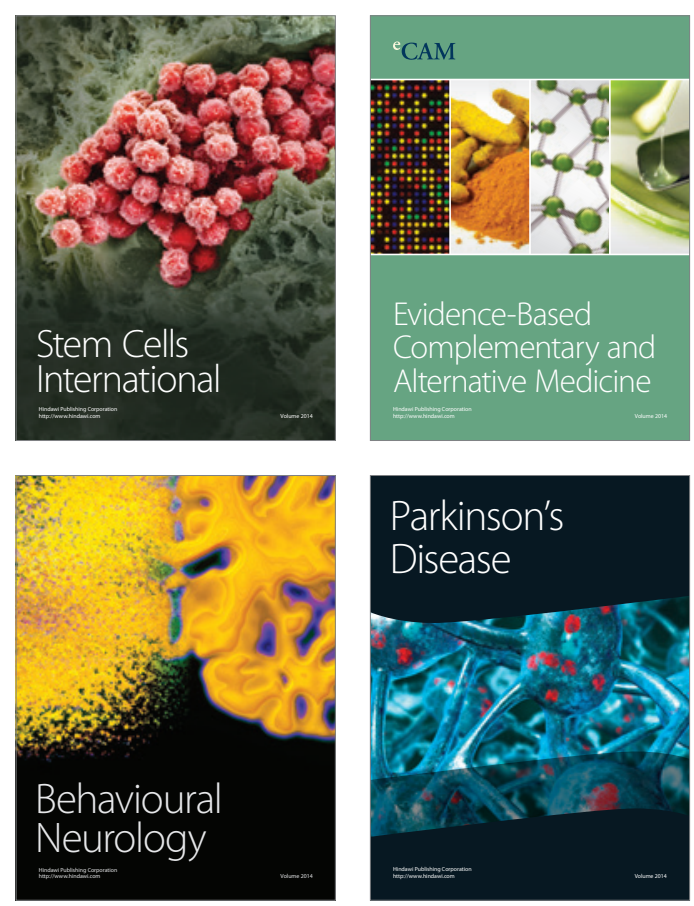

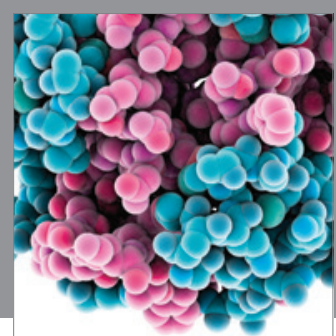

Journal of
Diabetes Research

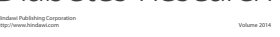

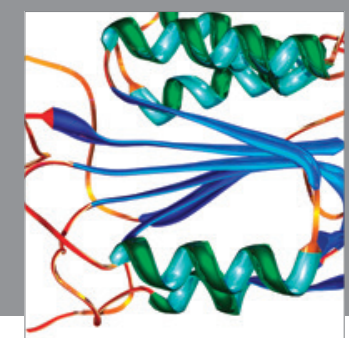

Disease Markers
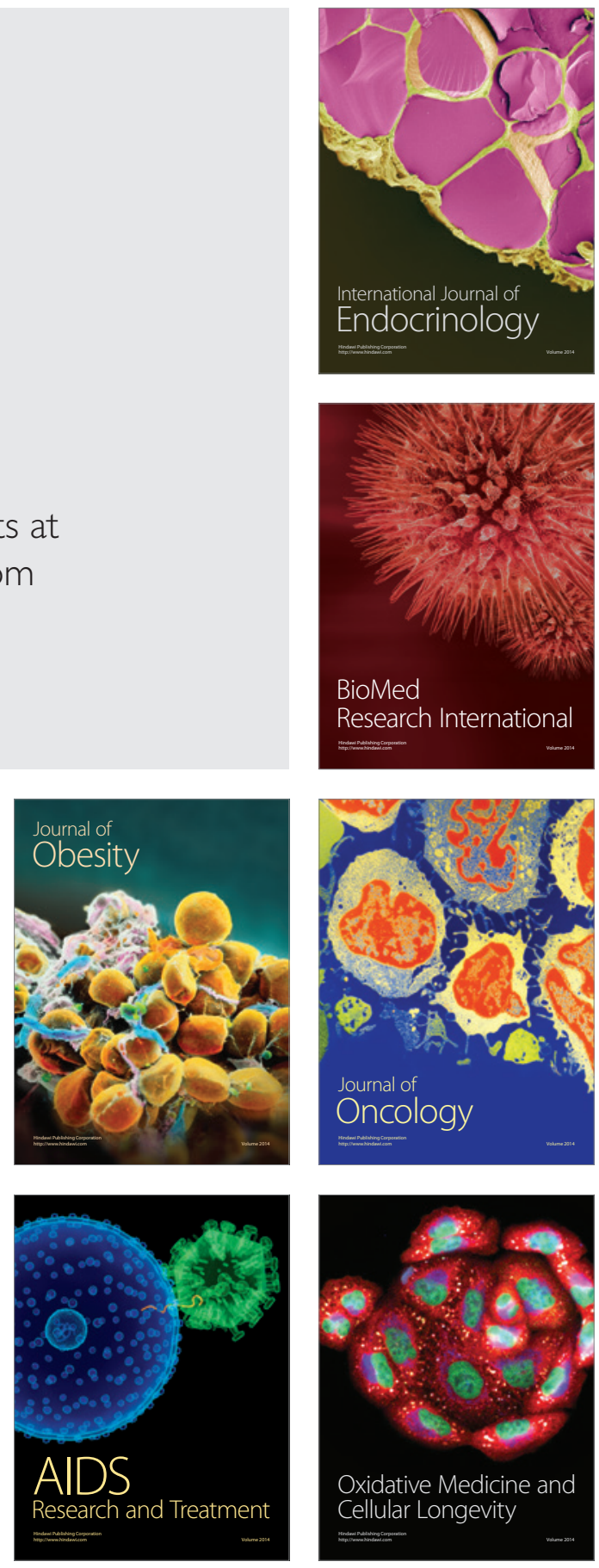